\title{
Establishing the correct management in a patient with multiple cryptogenic strokes: Antiplatelet vs. anticoagulant therapy
}

\author{
Ioan-Cristian Lupescu' ${ }^{1}$ Ioana Gabriela Lupescu ${ }^{2,3}$, Adriana Octaviana Dulamea ${ }^{1,3}$ \\ ${ }^{1}$ Neurology Clinic, Fundeni Clinical Institute, Bucharest, Romania \\ ${ }^{2}$ Radiology and Medical Imaging Department, Fundeni Clinical Institute, Bucharest, Romania \\ ${ }^{3}$ "Carol Davila" University of Medicine and Pharmacy, Bucharest, Romania
}

\begin{abstract}
Cryptogenic strokes represent a category of ischemic strokes in which there is no well-defined cause identified. For that matter, choosing between antiplatelet and anticoagulant therapy can be challenging. We present the case of a 44 year old Caucasian male with a history of multiple ischemic strokes, with thrombophilia, but no atrial fibrillation or known cardio-embolic sources, who initially received anticoagulant treatment, which was switched in 2015 with Clopidogrel. Aspirin was added in 2017 after having a TIA in the left MCA territory. Despite dual antiplatelet therapy, the patient developed ischemic stroke in the left MCA territory, for which re-initiation of anticoagulant treatment was decided. Holter ECG, transthoracic and transesophageal echocardiography were performed, but failed to identify a cause.
\end{abstract}

Keywords: cryptogenic stroke, thrombophilia, antiplatelet, anticoagulant

$\begin{array}{llll}\text { Abbreviations (in alphabetical order): } & \\ \text { ACA } & \text { anterior cerebral artery } & \text { MCA } & \text { middle cerebral artery } \\ \text { BMI } & \text { body mass index } & \text { MIP } & \text { maximum intensity projection } \\ \text { BP } & \text { blood pressure } & \text { MRI } & \text { magnetic resonance imaging } \\ \text { CT } & \text { computer tomography } & \text { mRS } & \text { modified Rankin Scale } \\ \text { DVT } & \text { deep venous thrombosis } & \text { MTHFR } & \text { methylene tetrahydrofolate reductase } \\ \text { ECG } & \text { electrocardiogram } & \text { NECT } & \text { non-enhanced computer tomography } \\ \text { EF } & \text { ejection fraction } & \text { TIA } & \text { transient ischemic attack } \\ \text { FSE } & \text { fast spin echo } & \text { TOF } & \text { Time-of-Flight } \\ \text { GRE } & \text { gradient echo } & \text { VSD } & \text { ventricular septal defect } \\ \text { HR } & \text { heart rate } & & \end{array}$

\section{INTRODUCTION}

Cryptogenic strokes represent a category of ischemic strokes in which no well-defined cause is identified, despite a thorough evaluation. It is believed that most of them are embolic in nature. Emphasis should be made on detection of occult paroxysmal atrial fibrillation (1), atheromatous aortic disease (2), hypercoagulable states and causes of paradoxical embolism (e.g. patent foramen ovale) (3). Management of patients may be difficult, be- cause of no clear underlying target. Antiplatelet therapy is usually recommended in non-cardioembolic strokes, while anticoagulant treatment is used in cases of positive screening for atrial fibrillation, regardless of its duration (4).

\section{CASE PRESENTATION}

We present the case of a 44 year old Caucasian male, non-smoker, casual alcohol consumer, with a history of multiple ischemic strokes (bilateral $A C A$ 
territory in 2010, left cerebellar and right parietal discovered on subsequent CT scans), mild cognitive impairment and also a history of seizures.

Medical history revealed VSD surgically closed at the age of 8, thrombophilia (homozygous MTHFR gene mutation, protein $\mathrm{S}$ deficiency, protein $\mathrm{C}$ deficiency), abdominal aortic aneurysm and hepatic hemangiomas.

Brain MRI done in 2015 revealed right temporal cavernoma, hypoplastic left vertebral artery and absence of the A1 segment of the right ACA, with origin of both post-communicating ACA segments from the left ACA (anatomical variant - Fig. 1).

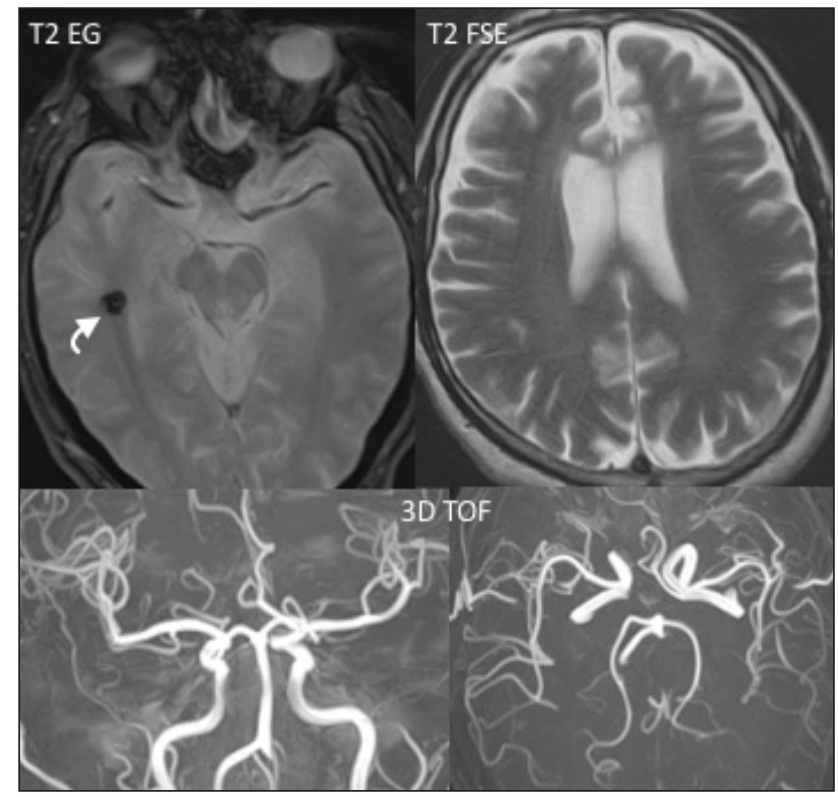

FIGURE 1. MRI evaluation in T2 GRE, T2 FSE and $3 D$-TOF with MIP reconstruction in coronal and axial planes: small hypointense right temporal nodule corresponding to hemosiderin into a cavernoma; moderate cerebral atrophy; hypoplastic left vertebral artery and absence of visualization of the A1 segment of the right $A C A$.

The patient initially received anticoagulant therapy and was switched on antiplatelet treatment in 2015 (Clopidogrel 75 mg q.d.).

In January 2017, the patient was admitted to the hospital for faintness, right hemiparesis and right-sided numbness.

Neurological exam highlighted right hemiparesis 4/5 MRC, right-sided hypoesthesia, hypophonia and abulia. HR was $60 \mathrm{bpm}$ rhythmic, with a BP of $110 / 70 \mathrm{mmHg}$ in supine position and $110 / 80 \mathrm{mmHg}$ while standing up.

Brain CT revealed no acute ischemic or hemorrhagic lesions.
Doppler Ultrasound of carotid and vertebral arteries showed normal flow with no atheromatous plaques.

Chest X-ray, ECG and laboratory findings were within normal range.

Given the rapid improvement of neurological status, the episode was regarded as a TIA. Dual antiplatelet therapy (Aspirin $75 \mathrm{mg}$ q.d. and Clopidogrel $75 \mathrm{mg}$ q.d.) was thus chosen as treatment, until further investigations.

Upon returning according to the appointment, the patient developed acute onset of right hemiplegia and mixed aphasia. Suspecting an acute stroke in the left MCA territory, brain CT was immediately performed, but didn't show any new lesions.

Thrombolytic therapy (Alteplase) was therefore administered, with subsequent improvement of neurological status.

Brain CT repeated 24 hours after thrombolytic therapy showed a newly formed hypodense lesion in the left parietal lobe with extension to the ipsilateral insulo-opercular region (Fig. 2 - white arrow).

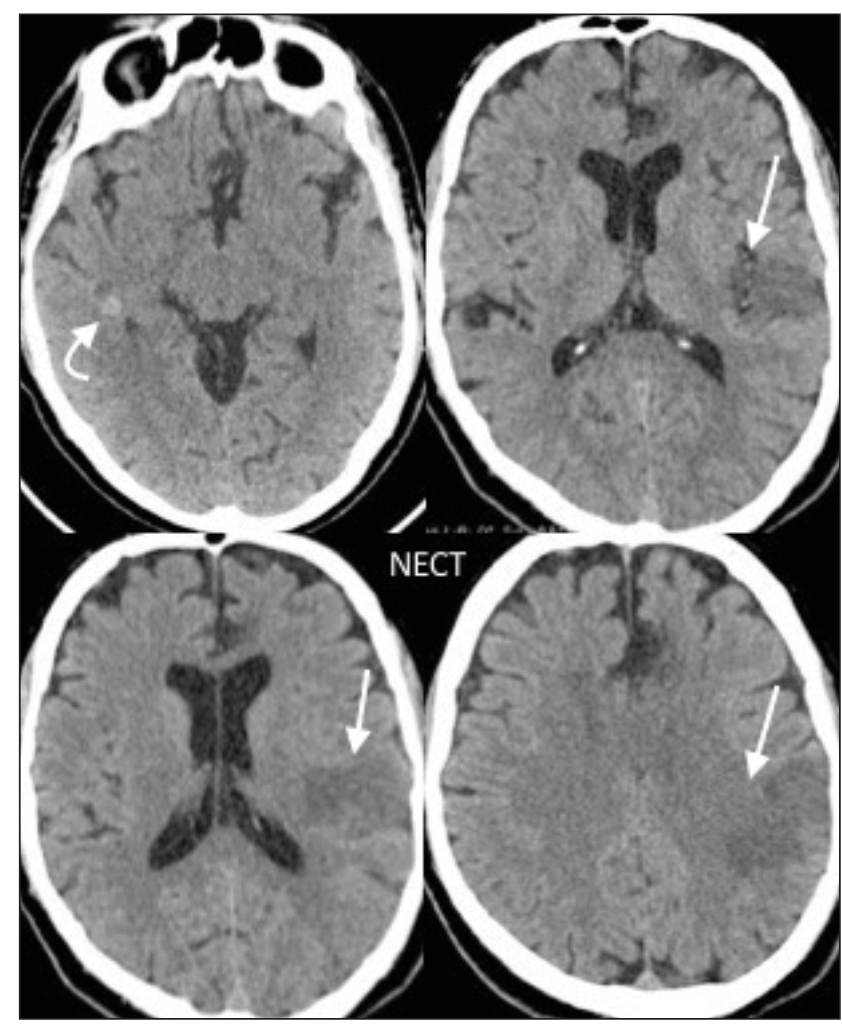

FIGURE 2. NECT showing the location of the left ischemic stroke (white arrow). The right temporal cavernoma appears as a round hyperdense lesion (curved arrow). There is cerebral atrophy more evident in the frontal regions.

Blood samples were collected, including coagulation, antiphospholipid antibodies and autoim- 
mune markers, all within normal range. Transthoracic echocardiography, brain MRI and Holter ECG were then performed for the purpose of detecting a cause for the strokes.

Transthoracic echocardiography failed to identify any of the well-known cardio-embolic sources. Considering the age of the patient, we then requested a transesophageal echocardiography, since an abnormal finding would have helped in establishing the correct management. (5)

TABLE 1. Holter ECG

\begin{tabular}{|l|}
\hline Sinus rhythm during whole monitoring \\
\hline Rare ventricular extrasystoles \\
\hline No sinus pauses over 2.5 seconds \\
\hline
\end{tabular}

TABLE 2. Transesophageal echocardiography

\begin{tabular}{|l|}
\hline Surgically closed VSD without shunt \\
\hline Intact interatrial septum \\
\hline Empty le auricle \\
\hline Aor c regurgita on grade I-II \\
\hline Normal right and le ventricular func ons \\
\hline
\end{tabular}

TABLE 3. Brain MRI

\begin{tabular}{|l|}
\hline Subacute ischemic stroke in le parieto-temporo-insular region \\
\hline Right temporal cavernoma with subacute hemorrhage \\
\hline Arteries of the Circle of Willis with homogenous signal \\
\hline Chronic ischemic strokes in the bilateral frontal regions \\
\hline
\end{tabular}

During hospital stay, antiplatelet therapy was discontinued and replaced with anticoagulant therapy.

The patient started neurological recovery (kinesiotherapy) and was discharged with a mRS of 2 . He was left at home with Acenocoumarol 3 mg q.d.

\section{DISCUSSIONS}

In our patient, no signs of atrial dysfunction or atrial fibrillation were detected, no cardiac septal abnormalities and no atheromatous lesions, the only predisposing factor being his thrombophilia.

Thrombophilia alone is overestimated as etiology for stroke and a second risk factor should always be searched $(6,7)$.

Because the patient had no well-defined cardio-embolic source, antiplatelet therapy was preferred and replaced anticoagulation in 2015.

The existence of the right temporal cavernoma did not influence decision making, as antithrombotic therapy doesn't increase the risk of cavernoma-related bleeding (8).

However, in the setting of inherited thrombophilia and recurrent ischemic strokes of unknown origin, anticoagulant therapy can be considered (4).

Especially in the setting of a recurrent ischemic stroke in spite of double antiplatelet therapy, the use of anticoagulation is justified.

The possibility of an occult cardio-embolic source (e.g. occult atrial fibrillation) must also be assessed, and for that the patient will need longterm ECG monitoring.

ACKNOWLEDGEMENTS: No financial gain was obtained for this case presentation.

Conflict of interest: none declared

\section{REFERENCES}

1. Gladstone D.J., Spring M., Dorian P. et al. Atrial fibrillation in patients with cryptogenic stroke. N Engl J Med. 2014; 370(26):2467-2477.

2. Amarenco P., Cohen A., Tzourio C. et al. Atherosclerotic disease of the aortic arch and the risk of ischemic stroke. N Engl J Med. 1994; 331(22):1474-1479.

3. Overell J.R., Bone I., Lees K.R. Interatrial septal abnormalities and stroke: a meta-analysis of case-control studies. Neurology. 2000; 55(8):1172-1179.

4. Kernan W.N., Ovbiagele B., Black H.R. et al. Guidelines for the prevention of stroke in patients with stroke and transient ischemic attack. Stroke. 2014; 45(7):2160-2236.

5. Katsanos A.H., Bhole R., Frogoudaki A. et al. The value of transesophageal echocardiography for embolic strokes of undetermined source. Neurology. 2016; 87(10):988-995.

6. Morris J.G., Singh S., Fisher M. Testing for inherited thrombophilias in arterial stroke: can it cause more harm than good? Stroke. 2010; 41(12):2985-2990.

7. Austin H., Chimowitz M.I., Hill H.A. et al. Cryptogenic stroke in relation to genetic variation in clotting factors and other genetic polymorphisms among young men and women. Stroke. 2002; 33(12):2762-2768.

8. Schneble H.M., Soumare A., Hervé D. et al. Antithrombotic therapy and bleeding risk in a prospective cohort study of patients with cerebral cavernous malformations. Stroke. 2012; 43(12):3196-3199. 